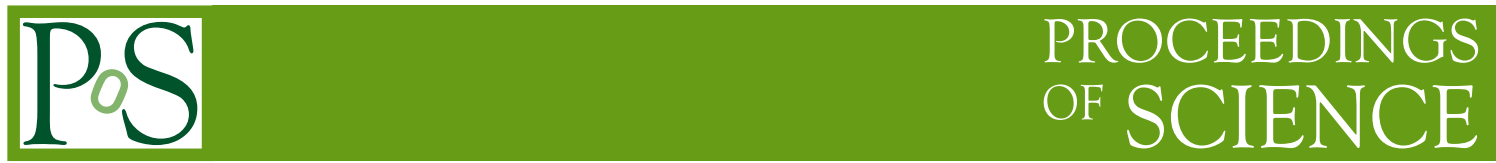

\title{
Top Quark Mass Measurements at the Tevatron
}

\author{
Reinhild Yvonne Peters ${ }^{* \dagger}$ \\ Georg-August Universität Göttingen, also at DESY \\ Friedrich-Hund-Platz 1 \\ 37077 Göttingen \\ Germany \\ E-mail: repeters@cern.ch
}

\begin{abstract}
Since the discovery of the top quark in 1995 by the CDF and D0 collaborations at the Fermilab Tevatron proton antiproton collider, precise measurements of its mass are ongoing. Using data recorded by the D0 and CDF experiment, corresponding to up to the full Tevatron data sample, top quark mass measurements performed in different final states using various extraction techniques are presented in this article. The recent Tevatron top quark mass combination yields $m_{t}=173.20 \pm 0.87 \mathrm{GeV}$. Furthermore, measurements of the top antitop quark mass difference from the Tevatron are discussed.
\end{abstract}

The European Physical Society Conference on High Energy Physics -EPS-HEP2013 18-24 July 2013

Stockholm, Sweden

\footnotetext{
* Speaker.

$\dagger$ on behalf of the CDF and D0 Collaborations.
} 


\section{Introduction}

In 1995, top quark was independently discovered by the CDF and D0 collaborations at the Tevatron proton antiproton $(p \bar{p})$ collider at Fermilab [1,2]. It is the heaviest known elementary particle. The top quark mass, $m_{t}$, is a free parameter in the standard model of particle physics (SM). Especially in the light of discovery of a Higgs boson by the ATLAS and CMS experiments at the LHC [3, 4], it is important to measure the top quark mass as precisely as possible and test its role in the mechanism of electroweak symmetry breaking.

Since the discovery of the top quark, several methods to measure the top quark mass have been developed and refined. Furthermore, various $t \bar{t}$ final states have been explored for these measurements. Thanks to dedicated methods and the large data samples of CDF and D0, precision measurements of the top quark mass in several channels exist by now. In order to provide the most precise number for the top quark mass, the individual measurements from both CDF and D0 were combined into one value.

In the following, the recent Tevatron combination of direct top quark mass measurements is presented, as well as recent results from the CDF and D0 collaborations that serve as inputs to the combination. In addition, an alternative approach to extract the top quark mass from the measurement of the $t \bar{t}$ cross section and measurements of the top antitop quark mass difference are discussed.

\section{Direct Top Quark Mass Measurements and Tevatron Combination}

The main production mechanism of top quarks at the Tevatron is via top quark pair production $(t \bar{t})$. In the SM, top quarks decay with almost $100 \%$ probability into a $b$ quark and a $W$ boson. The final states are classified according to the decays of the two $W$ bosons from $t \bar{t}$. The main channels are the dileptonic final state with both $W$ bosons decaying into leptons, the lepton+jets channel with one $W$ boson decaying into leptons and the other into a pair of quarks, and the all hadronic final state where both $W$ boson decays hadronically. Channels where at least one $W$ boson decays into a hadronically decaying tau-lepton are not considered in the analyses discussed here.

The recent combination of top quark mass measurements from the Tevatron uses results from Run I (1.8 TeV collision energy) and Run II (1.96 TeV collision energy) of the Tevatron in different final states and using different extraction methods, where data samples corresponding to an integrated luminosity of up to $8.7 \mathrm{fb}^{-1}$ were analyzed. The combination is performed using the Best Linear Unbiased Estimator (BLUE), taking into account correlations between systematic uncertainties. In the BLUE method, each input measurement receives a weight with which it contributes to the combination. The combined value of the top quark mass is $m_{t}=$ $173.20 \pm 0.51$ (stat) \pm 0.71 (syst) $\mathrm{GeV}$ [5]. This result is limited by systematic uncertainties, where the dominant ones arise from signal modeling and the calibration of the light jet energy scales. The $\chi^{2}$ of the combination is 8.5 for 11 degrees of freedom, which corresponds to a probability of $76 \%$ that the results are consistent between each other.

The result with the highest weight in the combination is the lepton+jets analysis by CDF, which uses the full Run II data sample of $8.7 \mathrm{fb}^{-1}$ [6]. In the lepton+jets final state one $W$ boson from top quark decays into a charged lepton and a neutrino, which leaves the detector without inter- 
acting. The other $W$ boson decays into a pair of quarks. The $t \bar{t}$ kinematics are reconstructed using a kinematic fit, where constraints from the known $W$ boson mass as well as the requirement that the reconstructed top and antitop masses are the same are included. An unbinned maximum likelihood fit is then performed using the reconstructed top quark mass $m_{t}^{\text {reco }}$ with lowest and second lowest $\chi^{2}$ value. To enhance the sensitivity, events with no, one or two identified $b$-jets are treated separately. The influence of the jet energy scale (JES) uncertainty on the result of the top quark mass measurement is reduced by fitting the JES in-situ using the two quarks from the $W$ boson decay and constraining their invariant mass to the knwon $W$ boson mass. The measured top quark mass, $m_{t}=172.85 \pm 0.71$ (stat) \pm 0.85 (syst) GeV representes the best single measurement to date. The systematic uncertainties are dominated by effects from the choice of Monte Carlo (MC) generator, color reconnection, the residual JES uncertainty and the $b$-jet energy scale.

The result with the second highest weight in the Tevatron combination is coming from an analysis in the lepton+jets final state by D0, using the matrix element (ME) method on $3.6 \mathrm{fb}^{-1}$ of Run II data [7]. The ME method explores the full kinematic information of each event by calculating per-event signal probabilities $P_{s i g}\left(x ; m_{t}\right)$ and background probablities $P_{b k g}(x)$, where $x$ denotes the momenta of the final state partons. The probabilities are calculated by integrating over the leading order (LO) matrix element for the $t \bar{t}$ production, folded with the parton distribution functions and transfer functions, which describe the transition of the parton momenta into the measured momenta of the final state particles from the top quark decays. The measured top quark mass is then obtained by maximizing the likelihood of the product of these per-event probabilities. Constraining the JES via the hadronically decaying $W$ boson, the measured value is $m_{t}=174.94 \pm$ 0.83 (stat) \pm 0.78 (JES) \pm 0.96 (syst) GeV. The dominant systematic uncertainties arise from the underlaying event and hadronization and color reconnection affecting the signal modeling, and from jet energy resolution and jet response uncertainties.

A new analysis by CDF using events with missing transverse energy $\left(Z_{T}\right)$ and jets on $8.7 \mathrm{fb}^{-1}$ of data represents the analysis having the third highest weight in the Tevatron combination. In this analysis, mainly lepton+jets events, where the electron or muon is not reconstructed, are recovered. The mass is extracted using templates of $m_{t}^{\text {reco }}$ with highest and second highest $\chi^{2}$, where the $\chi^{2}$ values are calculated using a modified kinematic fitter which only uses jets and the $\not_{T}$. The additional assumption in this kinematic fitter with respect to the kinematic fitter in lepton+jets events as used in [6] is that now both decay particles of the leptonically decaying $W$ boson are missing. Events with four, five or six jets are treated seprately, as are events with one or more than one identified $b$-jet. In events with five jets, $\tau+$ jets events are assumed, where the $\tau$ is misidentified as jet and the kinematic fitter is adjusted accordingly. The top quark mass yields $m_{t}=173.93 \pm$ 1.64 (stat) \pm 0.87 (syst) $\mathrm{GeV}$ [8], where the main contribution to the systematic uncertainties comes from effects on the residual JES and the signal modeling due to the choice of MC generator.

The next highest weight is provided by a measurement in the full hadronic final state by CDF, using $5.8 \mathrm{fb}^{-1}$ of data. For this analysis events with at least six jets, no leptons and no significant $B_{T}$ are used. Among the six leading jets, one or at least one have to be identified as $b$-jets. Templates of $m_{t}^{r e c o}$ are constructed via a $\chi^{2}$ like quantity. The two hadronically decaying $W$ bosons are used to constrain the JES. The main challenge in this analysis is the large background from QCD (quantum chromo dynamics) multijet events. The background consists of the QCD production of light and heavyflavor quarks, which is hard to simulate. Therefore this background is constructed using a 
data-driven method, which is based on the parametrization of the rate with which to identify a $b$ jet, derived in a sample of events with five jets that is dominated by background. Before extracting the mass, the sample is enriched in $t \bar{t}$ events, a neural network is constructed, that is based on variables that depend on the energy, direction and shape of the jets. The fitted top quark mass is $m_{t}=172.5 \pm 1.4$ (stat) \pm 1.4 (syst) $\mathrm{GeV}$ [9]. The main contribution to the systematic uncertainties arises from the choice of MC generator for the modeling of the signal, the construction of the QCD multijet background and uncertainties on the residual JES.

Another important contribution to the Tevatron combination is provided by the measurement of the top quark mass in the dileptonic final state, performed by D0 on $5.3 \mathrm{fb}^{-1}$ of data. The dilepton final state, where two isolated charged leptons (electron or muon) with large transverse momentum are required, has the advantage of being a very clean signature with small contribution from backgrounds. The main challenge is the more difficult reconstruction of the $t \bar{t}$ event from the final state objects, due to the presence of the two neutrinos. The latter results in an underconstrained system. At D0, two methods were explored in the recent measurement of the top quark mass, where once the ME method was explored [10], and in the other analysis the neutrino weighting method. The neutrino weighting technique uses assumptions about the $\eta^{1}$ of both neutrinos. For each neutrino $\eta$ sampling, solutions for the kinematics of the event are calculated, and a weight for each solution is assigned which is based on the agreement between the calculated neutrino transverse momenta and the measured value of the missing transverse energy. For the measurement of the top quark mass, the first two moments of the weight distribution are used to extract $m_{t}$. In addition, this analysis uses the measurement of the in-situ JES from the top quark mass measurement in the lepton+jets final state [7] to constrain the JES in the dilepton channel. This provides an improved JES calibration in the analysis. The $m_{t}$ measurements with both methods have been combined using the BLUE method, resulting in $m_{t}=173.9 \pm 1.9$ (stat) \pm 1.6(syst) GeV [11]. The main systematic uncertainties arise from uncertainties on the JES and the choice of MC generator for the modeling of the signal. Figure 1 (left) shows the different channels contributing to the combination as well as the combined value itself.

\section{Other Top Quark Mass related Measurements}

The methods discussed in the previous section of direct top quark mass measurements rely on MC simulation. Until today, it is under theoretical investigation how the measured top quark mass from $\mathrm{MC}$ is related to top quark pole or $\overline{M S}$ mass. The $\mathrm{D} 0$ collaboration has performed a determination of the top quark mass from the measurement of the $t \bar{t}$ cross section using $5.3 \mathrm{fb}^{-1}$ [12], which allows an unambiguous interpretation of the top quark mass. In this analysis the measured $t \bar{t}$ cross section is compared to inclusive calculations as function of the top quark mass. Using the pole mass for the inclusive cross section calculations, D0 extracted a pole mass of $m_{t}=167.5_{-4.7}^{+5.2} \mathrm{GeV}$ for the cross section calculation from Ref. [13], compatible with the top quark mass value from the Tevatron combination. Doing the same extraction again but with a calculation in the $\overline{M S}$ mass scheme yields about $7 \mathrm{GeV}$ smaller values for $m_{t}$. Figure 1 (right) shows the measured versus calculated $t \bar{t}$ cross section as function of the top quark mass.

\footnotetext{
${ }^{1}$ The rapidity $y$ and pseudorapidity $\eta$ are defined as functions of the polar angle $\theta$ and parameter $\beta$ as $y(\theta, \beta) \equiv$ $\frac{1}{2} \ln [(1+\beta \cos \theta) /(1-\beta \cos \theta)]$ and $\eta(\theta) \equiv y(\theta, 1)$, where $\beta$ is the ratio of a particle's momentum to its energy.
} 

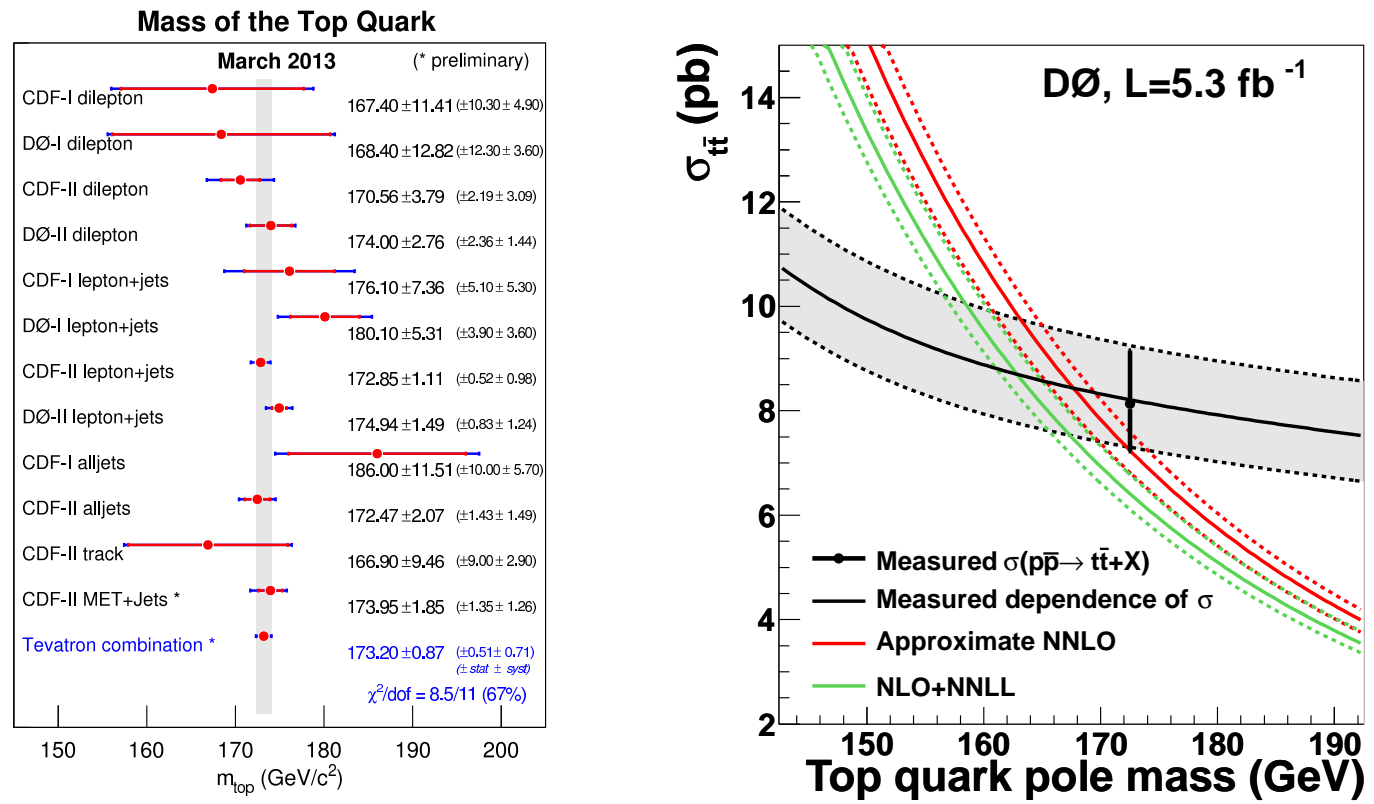

Figure 1: Recent Tevatron combination of top quark mass measurements from CDF and D0 (left) [5] and top quark mass extraction from the $t \bar{t}$ cross section (right) [12].

The direct top quark mass measurements assume the top and the antitop to be equally heavy. A mass difference between particle and its antiparticle would indicate CPT violation. The D0 and CDF collaborations have performed measurements of the top antitop quark mass difference. The first measurement of the mass difference between a bare quark and its antiquark was performed by the D0 collaboration using the ME method using $1 \mathrm{fb}^{-1}$ of data in the lepton+jets final state [14]. By repeating the measurement on $3.6 \mathrm{fb}^{-1}$ of data, D0 has extracted $m_{t}-m_{\bar{t}}=0.8 \pm 1.8$ (stat) \pm 0.5 (syst) $\mathrm{GeV}$ [15]. The CDF collaboration performed the mass difference measurement using a template technique in the lepton+jets channel, first using $5.6 \mathrm{fb}^{-1}$ of data [16], and then repeating the same measurement on the full Tevatron data sample of $8.7 \mathrm{fb}^{-1}$, resulting in $m_{t}-m_{\bar{t}}=-1.95 \pm$ 1.26 (stat + syst) $\mathrm{GeV}$ [17]. All results are compatible with the SM prediction and are limited by the statistical uncertainty.

\section{Conclusion and Outlook}

The measurement of the top quark mass is one of the legacy measurements at the Tevatron. The most recent top quark mass combination from the Tevatron has been presented in this article, as well as recent analyses in various channels that serve as input to the combination. Furthermore, the determination of the top quark mass from the $t \bar{t}$ cross section and measurements of the top antitop quark mass difference have been discussed. Some of the presented measurements already use the full Tevatron data sample. Currently, many different analyses in various channels are still ongoing to perform the final top quark mass measurements at D0 and CDF. Besides analysing the full data sample, the main challenge for top quark mass measurements at the Tevatron and the LHC is the understanding of the dominant sources of systematic uncertainty, mainly involving studies 
on the modeling of the $t \bar{t}$ signal. Given the importance of the top quark mass for the understanding of the SM, in particular in the context of electroweak symmetry breaking, the precise measurement of the top quark mass using various methods will stay an interesting field of study at all collider experiments.

\section{References}

[1] F. Abe et al. [CDF Collaboration], Phys. Rev. Lett. 74, 2626 (1995).

[2] S. Abachi et al. [D0 Collaboration], Phys. Rev. Lett. 74, 2632 (1995).

[3] The ATLAS Collaboration, Phys.Lett. B 716, 1 (2012).

[4] The CMS Colaboration, Phys. Lett. B 716, 30 (2012).

[5] The CDF and D0 Collaborations, arXiv:1305.3929 (2013).

[6] T. Aaltonen et al. [CDF Collaboration], Phys. Rev. Lett. 109, 152003 (2012).

[7] V. M. Abazov et al. [D0 Collaboration], Phys. Rev. D 84, 032004 (2011).

[8] Phys. Rev. D 88, 011101 (2013).

[9] The CDF Collaboration, Conf-note 10456 (2011).

[10] V. M. Abazov et al. [D0 Collaboration], Phys. Rev. Lett. 107, 082004 (2011).

[11] V. M. Abazov et al. [D0 Collaboration], Phys. Rev. D (RC) 86, 051103 (2012).

[12] V. M. Abazov et al. [D0 Collaboration], Phys. Lett. B 703, 422-427 (2011).

[13] S. Moch and P. Uwer, Phys. Rev. D 78, 034003 (2008); U. Langenfeld, S. Moch, and P. Uwer, Phys. Rev. D 80, 054009 (2009).

[14] V. M. Abazov et al. [D0 Collaboration], Phys. Rev. Lett. 103, 132001 (2009).

[15] V. M. Abazov et al. [D0 Collaboration], Phys. Rev. D 84, 052005 (2011).

[16] T. Aaltonen et al. [CDF Collaboration], Phys. Rev. Lett. 106, 161801 (2011).

[17] T. Aaltonen et al. [CDF Collaboration], Phys. Rev. D 87, 052013 (2013). 